\title{
Evaluating the performance of DFT methods in electric property calculations: sodium chloride as a test case
}

\section{George Maroulis}

Department of Chemistry, University of Patras, Patras, Greece

Correspondence: George Maroulis Department of Chemistry, University of Patras, 26504 Rio Patras, Greece Email maroulis@upatras.gr
This article was published in the following Dove Press journal:

Reports in Theoretical Chemistry

10 July 2013

Number of times this article has been viewed

\begin{abstract}
We present a quantitative analysis of the performance of conventional ab initio and density functional theory (DFT) methods. The important $\mathrm{NaCl}$ diatomic is chosen as testing ground. The analysis relies on metric considerations and pattern recognition techniques. Taking into account a collection of ab initio methods $\mathrm{A}=\{\mathrm{SCF}, \mathrm{MP} 2, \mathrm{CCSD}\}$ and DFT $=\{\mathrm{B} 3 \mathrm{LYP}$, B3PW91, mPW1PW91, mPW1PBE, PBEPW91, BHandH, BHandHLYP, HCTC , we find that the DFT method most close to CCSD is BHandHLYP. The DFT method closest to the collection of ab initio methods is BHandHLYP.
\end{abstract}

Keywords: ab initio, density functional theory, electric properties

\section{Introduction}

Density functional theory (DFT) based approaches offer an attractively economical (DFT) based alternative to the computationally more demanding conventional ab initio quantum chemical methods. ${ }^{1}$ The choice of a suitable DFT method is a matter of some importance and has been closely examined in authoritative reviews. ${ }^{2}$ Numerous systematic studies on the performance of DFT methods have been reported in recent years. $^{3-5}$ The relative merit of ab initio and DFT methods is not fully documented for many classes of molecular properties.

In previous work, we presented a theoretical method for the quantitative analysis of the performance of computational quantum methods. It is based on generalized metrics and pattern-recognition techniques. ${ }^{6}$ It has been applied to the rigorous analysis of the computational results obtained for the molecular properties of various systems. ${ }^{7-10}$ This method allows the introduction of order and classification in a space of theoretical descriptions of molecular systems. A theoretical description, associated with a welldefined quantum chemical method, is a collection of calculated values for an arbitrary set of molecular properties.

The properties of alkali halide clusters, and sodium chloride in particular, have attracted considerable attention in recent years. We mention only a selection of important papers here. Johnson et $\mathrm{al}^{11}$ and Ching et $\mathrm{al}^{12}$ reported linear and nonlinear susceptibilities of $\mathrm{NaCl}$ crystals relying on the local density approximation. The polarizability of the dimer $(\mathrm{NaCl})_{2}$ has been measured via molecular beam electric deflection by Guella et al. ${ }^{13}$ An analysis of the dipole polarizability of $(\mathrm{NaCl})_{2}$ was reported by Chauhan et al. ${ }^{14}$ Weis et $\mathrm{al}^{15}$ reported calculations of the dipole moments and polarizabilities of small stoichiometric $(\mathrm{NaCl})_{\mathrm{n}}$ and nonstoichiometric $\mathrm{Na}_{\mathrm{n}} \mathrm{Cl}_{\mathrm{n}-1}$ $\mathrm{n} \leq 4$ clusters. 
Our calculations in this paper pertain to $\mathrm{NaCl}$. The properties of interest in this work are the electric multipole moments dipole $(\mu)$, quadrupole $(\Theta)$, octopole $(\Omega)$, hexadecapole $(\Phi)$, dipole polarizability $(\alpha)$, and first dipole hyperpolarizability $(\beta)$. We are not aware of previous ab initio or DFT results for the higher electric moments of $\mathrm{NaCl}$.

\section{Theory}

Our approach to the calculation of electric properties has been presented in sufficient detail in previous work. ${ }^{16-20}$ We give only a few essentials here. The energy $\left(E^{p}\right)$ of an uncharged molecule interacting with a weak, static electric field can be written as an expansion: ${ }^{21,22}$

$E^{p} \equiv E^{p}\left(F_{\alpha}, F_{\alpha \beta}, F_{\alpha \beta \gamma}, F_{\alpha \beta \gamma \delta}, \ldots\right)$

$=\mathbf{E}^{0}-\mu_{\alpha} \mathrm{F}_{\alpha}-(1 / 3) \Theta_{\alpha \beta} \mathrm{F}_{\alpha \beta}-(1 / 15) \boldsymbol{\Omega}_{\alpha \beta \gamma} \mathrm{F}_{\alpha \beta \gamma}$

$-(1 / 105) \Phi_{\alpha \beta \gamma \delta} \mathrm{F}_{\alpha \beta \gamma \delta}+\cdots$

$-(1 / 2) \alpha_{\alpha \beta} F_{\alpha} F_{\beta}-(1 / 3) \mathbf{A}_{\alpha, \beta \gamma} F_{\alpha} F_{\beta \gamma}-(1 / 6) C_{\alpha \beta, \gamma \delta} F_{\alpha \beta} F_{\gamma \delta}$

$-(1 / 15) \mathbf{E}_{\alpha, \beta \gamma \delta} \mathrm{F}_{\alpha} \mathrm{F}_{\beta \gamma \delta}+\cdots-(1 / 6) \beta_{\alpha \beta \gamma} \mathrm{F}_{\alpha} \mathrm{F}_{\beta} \mathrm{F}_{\gamma}$

$-(1 / 6) B_{\alpha \beta, \gamma \delta} \mathrm{F}_{\alpha} \mathrm{F}_{\beta} \mathrm{F}_{\gamma \delta}+\cdots-(1 / 24) \gamma_{\alpha \beta \gamma \delta} \mathrm{F}_{\alpha} \mathrm{F}_{\beta} \mathrm{F}_{\gamma} \mathrm{F}_{\delta}+\cdots$

where $\mathrm{F}_{\alpha}, \mathrm{F}_{\alpha \beta}, \mathrm{F}_{\alpha \beta \gamma}$, etc, are the field, field gradient, etc at the origin of the molecule. The terms in bold are the permanent properties of the system: energy $\left(\mathrm{E}^{0}\right)$, multipole moments $\left(\mu_{\alpha}\right.$, $\left.\Theta_{\alpha \beta}, \Omega_{\alpha \beta \gamma}, \Phi_{\alpha \beta \gamma \delta}\right)$, polarizabilities $\left(\alpha_{\alpha \beta}, \mathrm{A}_{\alpha, \beta \gamma}, \mathrm{C}_{\alpha \beta, \gamma \delta}, \mathrm{E}_{\alpha, \beta \gamma \delta}\right)$, and hyperpolarizabilities $\left(\beta_{\alpha \beta \gamma}, \mathrm{B}_{\alpha \beta, \gamma \delta}, \gamma_{\alpha \beta \gamma \delta}\right)$. The subscripts denote Cartesian components. A repeated subscript implies summation over $\mathrm{x}, \mathrm{y}$, and $\mathrm{z}$. The number of independent components needed to specify the above tensors is strictly regulated by symmetry. ${ }^{23}$ The properties of interest in this work are the multipole moments, dipole $\left(\mu_{\alpha}\right)$, quadrupole $\left(\Theta_{\alpha \beta}\right)$, octopole $\left(\Omega_{\alpha \beta \gamma}\right)$ and hexadecapole $\left(\Phi_{\alpha \beta \gamma \delta}\right)$, the dipole polarizability $\left(\alpha_{\alpha \beta}\right)$, and the first dipole hyperpolarizability $\left(\beta_{\alpha \beta \gamma}\right)$. There is only one independent component for any electric moment tensor. With $\mathrm{z}$ as the molecular axis, we specify the electric moments by $\mu \equiv \mu_{z}, \Theta \equiv \Theta_{z z}, \Omega \equiv \Omega_{z z z}$, and $\Phi \equiv \Phi_{z z z z}$. The independent components for the polarizability $\alpha_{\alpha \beta}$ are $\alpha_{z z}$ and $\alpha_{\mathrm{xx}}$ and for the hyperpolarizability $\beta_{\mathrm{zzz}}, \beta_{\mathrm{zxx}}$. In addition to the above defined Cartesian components, we also calculate the following invariants:

$$
\begin{gathered}
\bar{\alpha}=\left(\alpha_{\mathrm{zz}}+2 \alpha_{\mathrm{xx}}\right) / 3 \\
\Delta \alpha=\alpha_{\mathrm{zz}}-\alpha_{\mathrm{xx}} \\
\bar{\beta}=(3 / 5)\left(\beta_{\mathrm{zzz}}+2 \beta_{\mathrm{zxx}}\right) \\
\Delta \beta=\beta_{\mathrm{zzz}}-3 \beta_{\mathrm{zxx}}
\end{gathered}
$$

We have used both $a b$ initio and DFT methods in this work. The ab initio methods are self-consistent-field (SCF), second-order Møller-Plesset perturbation theory (MP2), and singles and doubles coupled cluster (CCSD). Comprehensive presentations of these methods can be found in standard high-level textbooks. ${ }^{24-26}$ The DFT methods are B3LYP, B3PW9, mPW1PW91, mPW1PBE, PBEPW91, BHandH, BHandHLYP, and HCTH. A full documentation of these methods accompanies their implementation in the Gaussian 03 set of programs. ${ }^{27}$

\section{Proximity, similarity, and order in spaces of theoretical descriptions}

A comprehensive presentation of our methodology is fully presented in a recent paper. ${ }^{28}$ Only a few essential points are outlined here.

Let $\mathrm{TD}_{\mathrm{i}}$ be a collection of properties $\mathrm{Q}_{\mathrm{m} \alpha}$. The index $\mathrm{m}$ denotes methods and the index $\alpha$ denotes properties. The two indices take values in the index sets $\mathrm{I}_{\alpha}$ and $\mathrm{I}_{\mathrm{m}}$ :

$$
\mathrm{TD}_{\mathrm{i}}=\left(\mathrm{Q}_{\mathrm{m} \alpha}, \mathrm{m} \in \mathrm{I}_{\mathrm{m}}, \alpha \in \mathrm{I}_{\alpha}\right)
$$

The space of all theoretical descriptions $\mathrm{TD}_{\mathrm{i}}$ is denoted $\mathrm{TD}$,

$$
\mathrm{TD}=\left(\mathrm{TD}_{1}, \mathrm{TD}_{2}, \ldots, \mathrm{TD}_{\mathrm{N}}\right) \text { where } \mathrm{i}=1,2, \ldots, \mathrm{N}
$$

A generalized distance in the space of all TD is defined by using the Minkowski metric. Thus, the distance between two theoretical descriptions $\mathrm{TD}_{\mathrm{i}}$ and $\mathrm{TD}_{\mathrm{j}}$ is defined as:

$$
\begin{aligned}
D_{i j} & \equiv D\left(T_{i}, T D_{j}\right) \\
& =\left(\sum_{\alpha} \frac{\left|Q_{i \alpha}-Q_{j \alpha}\right|^{p}}{\left(\max _{i j}\left|Q_{i \alpha}-Q_{j \alpha}\right|\right)^{p}}\right)^{1 / p}, \quad p \geq 1,1 \leq i j \leq N
\end{aligned}
$$

In most applications, we have used the Euclidean metric, ie, $\mathrm{p}=2$.

Similarity between the two theoretical descriptions $\mathrm{TD}_{\mathrm{i}}$ and $\mathrm{TD}_{\mathrm{j}}$ is then defined on the basis of distance/proximity as:

$$
S_{i j}=1-\frac{D_{i j}}{\max _{i j} D_{i j}}, \quad 1 \leq i, j \leq N
$$

By definition, $0 \leq \mathrm{S}_{\mathrm{ij}} \leq 1$.

A connection with basic graph theory ${ }^{29}$ is established as follows. A graph $\mathrm{G}$ is a finite nonempty set of objects called vertices together with a set of unordered pairs of vertices called edges. The vertex set of $\mathrm{G}$ is denoted $\mathrm{V}(\mathrm{G})$ and the edge set $E(G)$. The cardinality p of $V(G)$ is called the order of 
$\mathrm{G}$ and the cardinality $\mathrm{q}$ of $\mathrm{E}(\mathrm{G})$ the size of $\mathrm{G}$. Let us consider the Cartesian product TD $\times$ TD. The graph of theoretical descriptions $\mathrm{G}_{\mathrm{TD}}$ has as vertex set $\mathrm{V}\left(\mathrm{G}_{\mathrm{TD}}\right)$ the set of theoretical descriptions $\mathrm{TD}_{\mathrm{i}}$. The edge set $\mathrm{E}\left(\mathrm{G}_{\mathrm{TD}}\right)$ is a subset of the Cartesian product defined above, $\mathrm{E}\left(\mathrm{G}_{\mathrm{TD}}\right) \subset \mathrm{TD} \times \mathrm{TD}$.

We assign to each edge of $\mathrm{G}_{\mathrm{TD}}$ a real, positive number, a weight. Consider the edge defined by $\mathrm{TD}_{\mathrm{i}}$ and $\mathrm{TD}_{\mathrm{j}}$. We assign to the edge $\left(\mathrm{TD}_{\mathrm{i}}, \mathrm{TD}_{\mathrm{j}}\right)$ the distance $\mathrm{D}_{\mathrm{ij}} \equiv \mathrm{D}\left(\mathrm{TD}_{\mathrm{i}}, \mathrm{TD}_{\mathrm{j}}\right) . \mathrm{G}_{\mathrm{TD}}$ is now a weighted graph.

The diameter Diam $\mathrm{G}_{\mathrm{TD}}$ of the graph of TD is defined as:

Diam $\mathrm{G}_{\mathrm{TD}}=\max _{\mathrm{i}, \mathrm{j} \in \mathrm{V}\left(\mathrm{G}_{\mathrm{TD}}\right)} \mathrm{D}_{\mathrm{ij}} \equiv \max _{\mathrm{TD}_{\mathrm{i}}, \mathrm{TD}_{\mathrm{j}} \in \mathrm{V}\left(\mathrm{G}_{\mathrm{TD}}\right)} \mathrm{D}\left(\mathrm{TD}_{\mathrm{i}}, \mathrm{TD}_{\mathrm{j}}\right)$

The similarity of $\mathrm{TD}_{\mathrm{i}}$ to $\mathrm{TD}_{\mathrm{j}}$ can now be written as:

$\mathrm{S}_{\mathrm{ij}} \equiv \mathrm{S}\left(\mathrm{TD}_{\mathrm{i}}, \mathrm{TD}_{\mathrm{j}}\right)=1-\frac{\mathrm{D}\left(\mathrm{TD}_{\mathrm{i}}, \mathrm{TD}_{\mathrm{j}}\right)}{\operatorname{Diam} \mathrm{G}_{\mathrm{TD}}}, \mathrm{TD}_{\mathrm{i}}, \mathrm{TD}_{\mathrm{j}} \in \mathrm{V}\left(\mathrm{G}_{\mathrm{TD}}\right)$

The distance of a fixed vertex $u$ from a subset of the vertex set $\mathrm{S} \subseteq \mathrm{V}\left(\mathrm{G}_{\mathrm{TD}}\right)$ is defined as:

$$
\mathrm{D}(\mathrm{u}, S)=\min _{\mathrm{x} \in \mathrm{S}}\{\mathrm{D}(\mathrm{u}, \mathrm{x})\}
$$

A point of major importance to our methodology is the definition of a minimum spanning tree (MST). A spanning subgraph $H$ of a graph $G$ has vertex and edge sets $V(H) \subseteq$ $\mathrm{V}(\mathrm{G})$ and $\mathrm{E}(\mathrm{H}) \subseteq \mathrm{E}(\mathrm{G})$ and is of the same order as $\mathrm{G}$. A graph $\mathrm{G}$ of order $\mathrm{p}$ and size $\mathrm{q}$ is a tree if and only if it is acyclic and $\mathrm{p}=\mathrm{q}+1$. The weight of a spanning tree in a connected graph is the sum of the weights of its edges. Thus an MST of G is a spanning tree of $\mathrm{G}$ of minimum weight. Suitable algorithms are available for the construction of an $\mathrm{MST} .{ }^{30}$ On the basis of the MST, we further perform a single-linkage cluster analysis by removing all edges from the MST characterized by weights above a given threshold $\mathrm{D}_{\mathrm{T}}$.

This analysis creates a partition of the MST in distinct clusters: $\mathrm{C}_{1}, \mathrm{C}_{2}, \ldots, \mathrm{C}_{\mathrm{K}}$. The union of all clusters is the vertex set of the $\mathrm{G}_{\mathrm{TD}}$ graph: $\mathrm{V}\left(\mathrm{G}_{\mathrm{TD}}\right)=\bigcup_{1 \leq i \leq K} C_{i}$. Last, we define the nearest-neighbor distance between clusters $\mathrm{C}_{\mathrm{m}}$ and $\mathrm{C}_{\mathrm{n}}$ as:

$$
\mathrm{D}_{\mathrm{nn}}\left(\mathrm{C}_{\mathrm{m}}, \mathrm{C}_{\mathrm{n}}\right)=\min _{\mathrm{TD}_{\mathrm{i}} \in \mathrm{C}_{\mathrm{m}}, \mathrm{TD}_{\mathrm{j}} \in \mathrm{C}_{\mathrm{n}}} \mathrm{D}\left(\mathrm{TD}_{\mathrm{i}}, \mathrm{TD}_{\mathrm{j}}\right)
$$

\section{Computational details}

All calculations in this work were performed with the large aug-cc-pvtz basis set, ${ }^{31}$ as implemented in Gaussian 03. For $\mathrm{NaCl}$, this basis set in contracted form is of [6s5p3d2f] type and consists of 100 contracted Gaussian-type functions.
All calculations were carried out at the experimental equilibrium bond length ${ }^{32}$ of $\mathrm{R}_{\mathrm{e}}=2.360795 \AA$. The molecule is placed on the $\mathrm{z}$-axis with the $\mathrm{Cl}$ center on its positive part and the center on mass on the origin $(0,0,0)$. The position of the atomic centers on the $\mathrm{z}$-axis is defined by $(0,0,-1.4243660256)$ for $\mathrm{Na}$ and $(0,0,0.9364289744)$ for $\mathrm{Cl}$.

In the MP2 and CCSD calculations, the ten innermost MO were kept frozen. All calculations were performed with Gaussian 03.

Unless otherwise specified, atomic units are used throughout this paper. Conversion factors to SI units are: energy, $1 \mathrm{E}_{\mathrm{h}}=4.3597482 \times 10^{-18} \mathrm{~J}$; length, $1 \mathrm{a}_{0}=0.529177249 \times 10^{-10} \mathrm{~m}$; $\mu, 1 \mathrm{ea}_{0}=8.478358 \times 10^{-30} \mathrm{~cm} ; \Theta, 1 \mathrm{ea}_{0}^{2}=4.486554 \times 10^{-40} \mathrm{~cm}^{2}$; $\Omega, 1 \mathrm{ea}_{0}{ }^{3}=2.374182 \times 10^{-50} \mathrm{~cm}^{3} ; \Phi, 1 \mathrm{ea}_{0}{ }^{4}=1.256363 \times$ $10^{-60} \mathrm{~cm}^{4} ; \alpha, 1 \mathrm{e}^{2} \mathrm{a}_{0}^{2} \mathrm{E}_{\mathrm{h}}^{-1}=1.648778 \times 10^{-41} \mathrm{c}^{2} \mathrm{~m}^{2} \mathrm{~J}^{-1}$; and $\beta$, $1 \mathrm{e}^{3} \mathrm{a}_{0}{ }^{3} \mathrm{E}_{\mathrm{h}}{ }^{-2}=3.206361 \times 10^{-53} \mathrm{c}^{3} \mathrm{~m}^{3} \mathrm{~J}^{-2}$. We give property values as pure numbers in most cases, ie, $\mu / \mathrm{ea}_{0}, \Theta / \mathrm{ea}_{0}{ }^{2}, \Omega / \mathrm{ea}_{0}{ }^{3}$, $\Phi / \mathrm{ea}_{0}^{4}, \alpha / \mathrm{e}^{2} \mathrm{a}_{0}^{2} \mathrm{E}_{\mathrm{h}}^{-1}, \beta / \mathrm{e}^{3} \mathrm{a}_{0}{ }^{3} \mathrm{E}_{\mathrm{h}}^{-2}$.

\section{Results and discussion}

The calculated dipole moments are shown in Table 1. Cartesian components and invariants of the dipole polarizability and first dipole hyperpolarizability are given in Tables 2 and 3, respectively. Last, in Table 4, we show the calculated similarities of the theoretical predictions obtained with the employed ab initio and DFT methods.

\section{Electric moments}

Our best values for the electric moments of $\mathrm{NaCl}$ are calculated at the CCSD level of theory and are: $\mu=-3.6212$, $\Theta=6.2549, \Omega=-20.30$, and $\Phi=50.33$. A comparison of the SCF values to those calculated at the MP2 and CCSD

Table I Electric multipole moments ${ }^{\mathrm{a}}$ for $\mathrm{NaCl}$ at the experimental bond length ${ }^{\mathrm{b}}$

\begin{tabular}{lllll}
\hline Method & $\mu$ & $\Theta$ & $\Omega$ & $\Phi$ \\
\hline SCF & -3.6710 & 6.1495 & -20.42 & 50.03 \\
MP2 & -3.6106 & 6.2727 & -20.28 & 50.47 \\
CCSD & $-\mathbf{3 . 6 2 1 2}$ & $\mathbf{6 . 2 5 4 9}$ & $-\mathbf{2 0 . 3 0}$ & $\mathbf{5 0 . 3 3}$ \\
B3LYP & -3.4546 & 6.1996 & -19.57 & 51.17 \\
B3PW9I & -3.5094 & 6.2544 & -19.85 & 51.22 \\
mPWIPW9I & -3.5069 & 6.2313 & -19.78 & 51.31 \\
mPWIPBE & -3.5067 & 6.2325 & -19.77 & 51.31 \\
PBEPW9I & -3.3779 & 6.2150 & -19.21 & 51.39 \\
BHandH & -3.5248 & 6.2497 & -19.99 & 51.44 \\
BHandHLYP & -3.5474 & 6.1871 & -20.00 & 50.88 \\
HCTH & -3.3805 & 6.0704 & -19.19 & 50.60 \\
\hline
\end{tabular}

Notes: Reference CCSD results in bold. ${ }^{\text {TT }}$ The higher electric moments $(\Theta, \Omega, \Phi)$ are relative to the center of mass; ${ }^{b} \mathbf{z}$ is the molecular axis with the $\mathrm{Cl}$ center on its positive part. 
Table 2 Electric dipole polarizability for $\mathrm{NaCl}$ at the experimental bond length

\begin{tabular}{lllll}
\hline Method & $\alpha_{\mathrm{xx}}$ & $\alpha_{\mathrm{zz}}$ & $\bar{\alpha}$ & $\Delta \boldsymbol{\alpha}$ \\
\hline SCF & 25.3739 & $27.968 I$ & 26.2386 & 2.5942 \\
MP2 & 28.1391 & $31.887 \mathrm{I}$ & 29.3884 & 3.7480 \\
CCSD & $\mathbf{2 7 . 5 9 4 8}$ & $\mathbf{3 0 . 9 6 8 I}$ & $\mathbf{2 8 . 7 1 9 2}$ & $\mathbf{3 . 3 7 3 4}$ \\
B3LYP & 30.1506 & 40.1523 & 33.4845 & 10.0016 \\
B3PW9I & 29.4038 & 36.5840 & 31.7972 & 7.1802 \\
mPWIPW9I & 29.0125 & 36.0368 & 31.3539 & 7.0244 \\
mPWIPBE & 29.0216 & 36.0315 & 31.3583 & 7.0098 \\
PBEPW9I & 32.1907 & 45.2993 & 36.5602 & 13.1085 \\
BHandH & 27.5908 & 33.7320 & 29.6378 & 6.1412 \\
BHandHLYP & 27.4395 & 33.6765 & 29.5185 & 6.2370 \\
HCTH & 31.8058 & 50.5204 & 38.0440 & 18.7146 \\
\hline
\end{tabular}

Note: Reference CCSD results in bold.

levels reveals a relatively small electron correlation effect. The CCSD value of $|\mu|$ is only $1.36 \%$ lower than the SCF value of -3.6710 . For the quadrupole moment, the SCF value is 6.1495. The CCSD result is just $1.71 \%$ above the SCF one. Small correlation effects are also observed for the octopole and hexadecapole moments. Finally, the MP2 values are quite close to the reference CCSD ones.

The calculated DFT values are convincingly close to presumably the most accurate CCSD results. For the dipole moment, the PBEPW91 method is the most distant from CCSD. It yields -3.3779 , a value lower (in absolute terms) than the CCSD by $6.72 \%$. For the quadrupole moment, the most distant from CCSD is the HCTH method. It yields 6.0704, just $2.89 \%$ less than the CCSD results.

\section{Polarizability}

The ab initio values for the polarizability show that electron correlation has a positive effect on the Cartesian components.

Table 3 Electric dipole hyperpolarizability for $\mathrm{NaCl}$ at the experimental bond length

\begin{tabular}{|c|c|c|c|c|}
\hline Method & $\beta_{z x x}$ & $\beta_{\mathrm{zzz}}$ & $\bar{\beta}$ & $\Delta \beta$ \\
\hline SCF & -93.25 & -259.51 & -267.60 & 20.22 \\
\hline MP2 & -142.80 & -427.21 & -427.68 & 1.17 \\
\hline CCSD & -132.28 & -381.48 & -387.62 & 15.35 \\
\hline B3 LYP & -208.15 & -1015.76 & -859.23 & -391.33 \\
\hline B3PW9I & -186.33 & -760.47 & -679.88 & -201.48 \\
\hline mPWIPW9I & -168.95 & -676.46 & -608.62 & -169.62 \\
\hline mPWIPBE & -169.06 & -676.22 & -608.60 & -169.04 \\
\hline PBEPW9I & -267.20 & -1386.44 & -|| $52.5 \mid$ & -584.83 \\
\hline BHandH & -140.80 & -558.70 & -504.19 & -136.28 \\
\hline BHandHLYP & -140.94 & -580.42 & -517.39 & -157.59 \\
\hline НСТH & -310.69 & -2322.94 & -1766.59 & -1390.88 \\
\hline
\end{tabular}

Note: Reference CCSD results in bold.

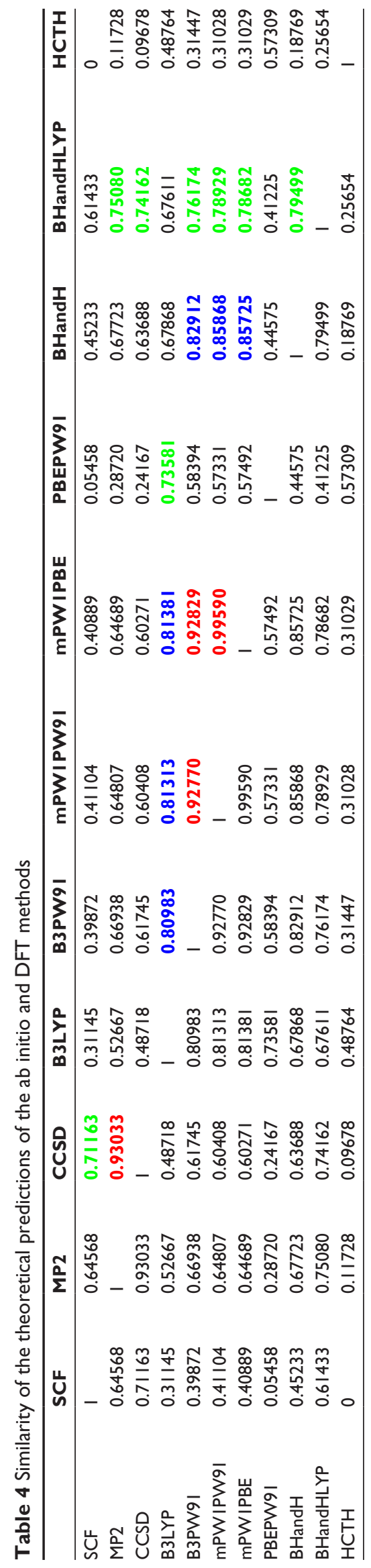

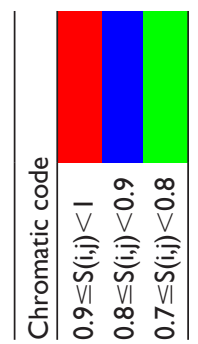


Our CCSD values are $\alpha_{\mathrm{xx}}=27.5948$ and $\alpha_{\mathrm{zz}}=30.9681$ These values are $8.75 \%$ and $10.73 \%$ higher, respectively, than the SCF values. The mean and the anisotropy at the CCSD level are $\bar{\alpha}=28.7192$ and $\Delta \alpha=3.3734$.

Substantial discrepancies are observed between the ab initio and DFT results. In Figure 1, we show the DFT-method dependence of the Cartesian components of $\mathrm{NaCl}$. The reference CCSD values are also shown. The PBEPW91 and HCTH methods are far from the CCSD. The two methods, $\mathrm{BHandH}$ and BHandHLYP, yield values that are closest to the ab initio values. The mean values calculated for the DFT methods are always above the ab initio. Most important, for the anisotropy, the DFT values are in some cases in net disagreement with the $\mathrm{ab}$ initio ones.

\section{Hyperpolarizability}

The SCF values of the hyperpolarizability are $\beta_{z x x}=-93.25$ and $\beta_{z z z}=-259.51$. Electron correlation has a very strong effect on these components. The CCSD values are significantly larger in magnitude at $\beta_{\mathrm{zxx}}=-132.28$ and $\beta_{\text {zzz }}=-381.48$, or $41.9 \%$ and $47.0 \%$, respectively. Strong method dependence is observed for the DFT results. The effect is especially pronounced for the longitudinal component. For $\beta_{z z z}$ the B3LYP, PBEPW91, and HCTH methods yield very large values. A very eloquent picture of this method dependence is shown in Figure 2. We note again the good performance of the BHandH and BHandHLYP methods for both Cartesian components. The extreme variation

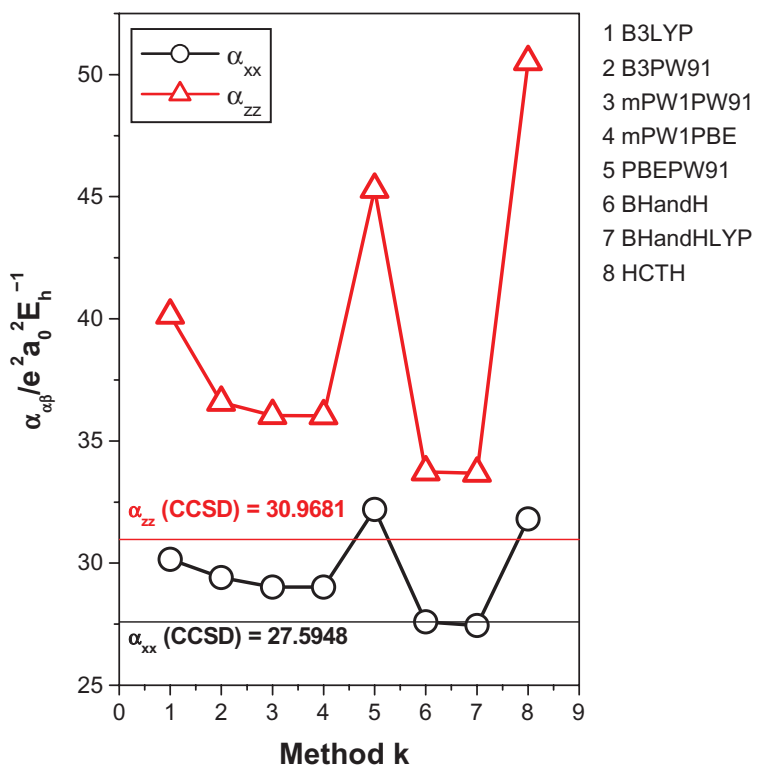

Figure I Method dependence of the DFT values of the dipole polarizability Cartesian components. Reference CCSD values also shown.

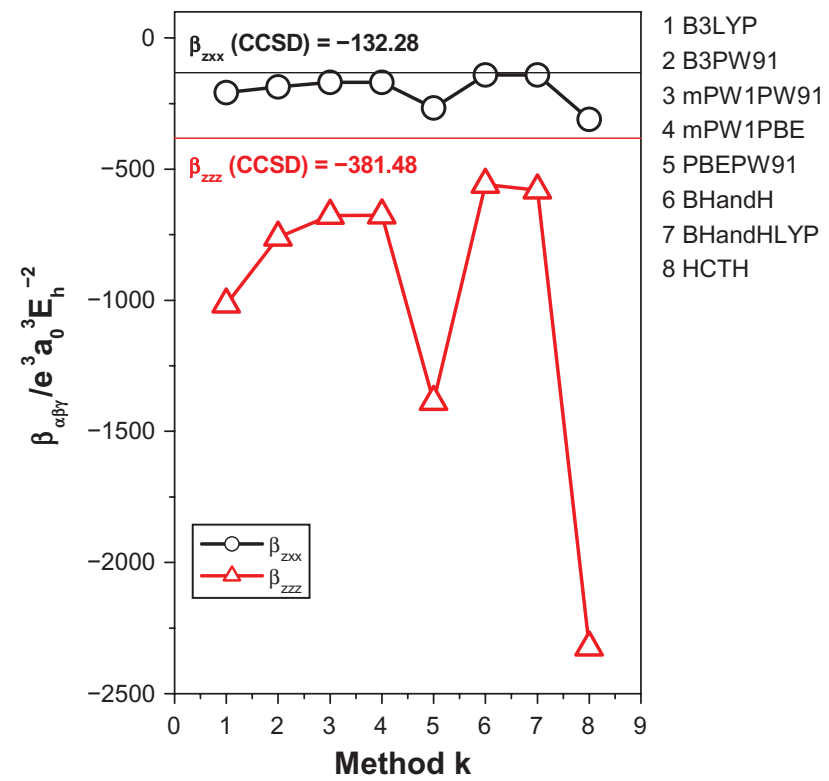

Figure 2 Method-dependence of the DFT values of the dipole hyperpolarizability Cartesian components. Reference CCSD values also shown.

of the hyperpolarizability results is easily brought forth by a comparison of the DFT hyperpolarizability invariants to the reference $\operatorname{CCSD}$ values of $\bar{\beta}=-387.62$ and $\Delta \beta=15.35$. In comparison, the $\mathrm{HCTH}$ values are considerably larger at $\bar{\beta}=-1766.59$ and $\Delta \beta=-1390.88$.

\section{Comparison with experiment and previous theoretical work}

A model study of the dipole moment (and other properties) of $\mathrm{NaCl}$ can be found in the early work of Matcha. ${ }^{33}$ We focus on more recent results. Bacskay and Buckingham ${ }^{34}$ calculated the dipole moment of $\mathrm{NaCl}$ using a (8s6p3d/9s7p4d) basis set. Their SCF and CCSD values were 3.6355 and 3.5457, respectively, in good agreement with ours. In his thorough theoretical investigation of the electric properties of alkali halides, Pluta $^{35}$ reported SCF, MP2, CCSD and CCSD(T) values for the dipole moment and (hyper)polarizability. His CCSD/HyPol values are (SCF results in parentheses) $\mu=3.549$ (3.642), $\alpha_{\mathrm{xx}}=28.88$ (25.85), $\alpha_{\mathrm{zz}}=33.21(28.45), \beta_{\mathrm{zxx}}=-114(-73)$ and $\beta_{z z z}=-412(-224)$. Our results are in essential agreement with Pluta's findings.

We are not aware of available experimental data for electric properties of $\mathrm{NaCl}$ other than the dipole. The equilibrium value of this property has been measured by the molecular beam electric resonance method by Hebert et al. ${ }^{36}$ Its value is relatively large $\left|\mu_{\mathrm{e}}(\mathrm{NaCl})\right|=3.53$ to be compared to that of the isoelectronic SiS molecule, $\left|\mu_{\mathrm{e}}(\mathrm{SiS})\right|=0.6099 .{ }^{37}$ 
Our CCSD result, $\mu_{\mathrm{e}}=-3.6212$, is reasonably close to the experimental value.

\section{Metrics and pattern recognition}

Our calculation of the proximity/similarity of the performance of the theoretical methods employed in this work take into account the electric moments and the Cartesian components of the (hyper)polarizability. Thus, the theoretical descriptions are defined by collections of numbers:

$$
\left(\mu, \Theta, \Omega, \Phi, \alpha_{x x}, \alpha_{z z}, \beta_{z x x}, \beta_{z z z}\right)
$$

Thus, our metric considerations pertain to points in an 8-D space. The calculated similarity quantities are given in Table 4 . In addition, we use a chromatic code in order to classify similarities in the region $0.7<\mathrm{S}(\mathrm{i}, \mathrm{j})<1.0$. In Figure 3 , we show a histogram of the similarities of all methods to presumably the most accurate one employed in this work. It is obvious that the performance of the MP2 method is quite close to that of the CCSD one, as attested by the high value of the similarity:

$$
\mathrm{S}(\mathrm{MP} 2, \mathrm{CCSD})=0.93033
$$

With the notable exception of PBEPW91 and HCTH, all other DFT methods are quite similar to ab initio. The DFT method most close to the latter is BHandHLYP. It is quite remarkable that this method is very similar to most of the

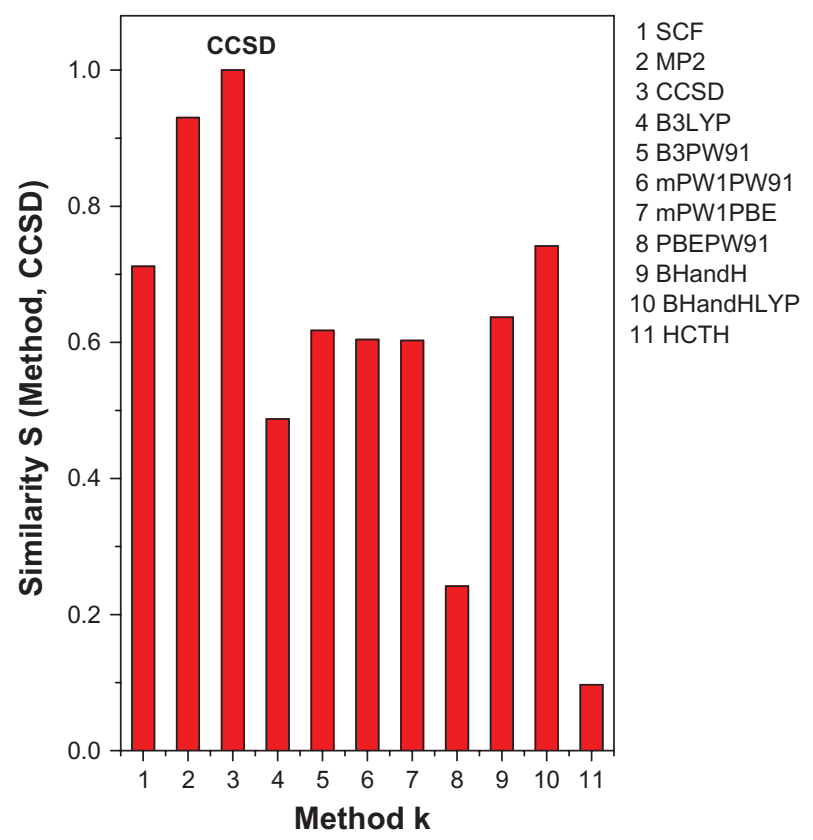

Figure 3 Histogram of the similarity of the ab initio and DFT methods to the reference CCSD description.
DFT of interest in this paper. This is easily brought forth by the pertinent entries in Table 4. Other interesting remarks concern the high similarity of the following pairs of DFT methods:

$$
\begin{gathered}
\mathrm{S}(\mathrm{mPW} 1 \mathrm{PW} 91, \mathrm{mPW} 1 \mathrm{PBE})=0.99590 \\
\mathrm{~S}(\mathrm{~B} 3 \mathrm{PW} 1, \mathrm{mPW} 1 \mathrm{PBE})=0.92829 \\
\mathrm{~S}(\mathrm{mPW} 1 \mathrm{PW} 1, \mathrm{~B} 3 \mathrm{PW} 91)=0.92770
\end{gathered}
$$

The performances of mPW1PW91 and mPW1PBE are virtually indistinguishable.

In Figure 4, we show the MST and the subsequent clustering by using two threshold distances, $\mathrm{D}_{\mathrm{T}}=0.5$ and 0.3 . The first clustering $\left(\mathrm{D}_{\mathrm{T}}=0.5\right)$ leads to the following partitioning of the TD space:

$$
\begin{aligned}
\mathrm{TD}= & \{\mathrm{SCF}\} \cup\{\mathrm{MP} 2, \mathrm{CCSD}\} \cup\{\mathrm{B} 3 \mathrm{LYP}, \mathrm{B} 3 \mathrm{PW} 91, \\
& \mathrm{mPW} 1 \mathrm{PW} 91, \mathrm{mPW} 1 \mathrm{PBE}, \mathrm{BHandH}\} \\
& \cup\{\mathrm{PBEPW} 1\} \cup\{\mathrm{BH} \text { andHLYP }\} \cup\{\mathrm{HCTH}\}
\end{aligned}
$$

Reducing the threshold distance to 0.3 , the large cluster decomposes further to:

(B3LYP, B3PW91, mPW1PW91, mPW1PBE, BHandH)

$=($ B3LYP $) \cup(B 3 P W 91, \mathrm{mPW} 1 \mathrm{PW} 91, \mathrm{mPW} 1 \mathrm{PBE})$

$\cup(\mathrm{BHandH})$

This shows that MP2, CCSD, and DFT B3PW91, mPW1PW91, and mPW1PBE display very similar performances compared to the other ab initio and DFT methods.

Last, in Figure 5, we show the variation of the distance $\mathrm{D}(\mathrm{k}, \mathrm{A})$, where:

$\mathrm{k} \in$ (B3LYP, B3PW91, mPW1PW91, mPW1PBE, PBEPW91, BHandH, BHandHLYP, HCTH) and A = (SCF, $\mathrm{MP} 2, \mathrm{CCSD})$. The graph in this figure shows the variation of the distance of the DFT methods from the subset of $a b$ initio ones. The conclusions drawn thereof are summarized as follows:

1. The most dissimilar DFT methods, as compared to the $\mathrm{ab}$ initio in set $\mathrm{A}$, are, in increasing distance, B3LYP, PBEPW91 and HCTH.

2. Methods B3PW91, mPW1PW91 and mPW1PBE are very close to ab initio.

3. The closest to the ab initio methods.

Succinctly,

$$
\mathrm{D}(\mathrm{HCTH}, \mathrm{A})>\underset{\mathrm{k} \in \mathrm{DFT}}{\mathrm{D}(\mathrm{k}, \mathrm{A})}>\mathrm{D}(\mathrm{BHandHLYP}, \mathrm{A})
$$



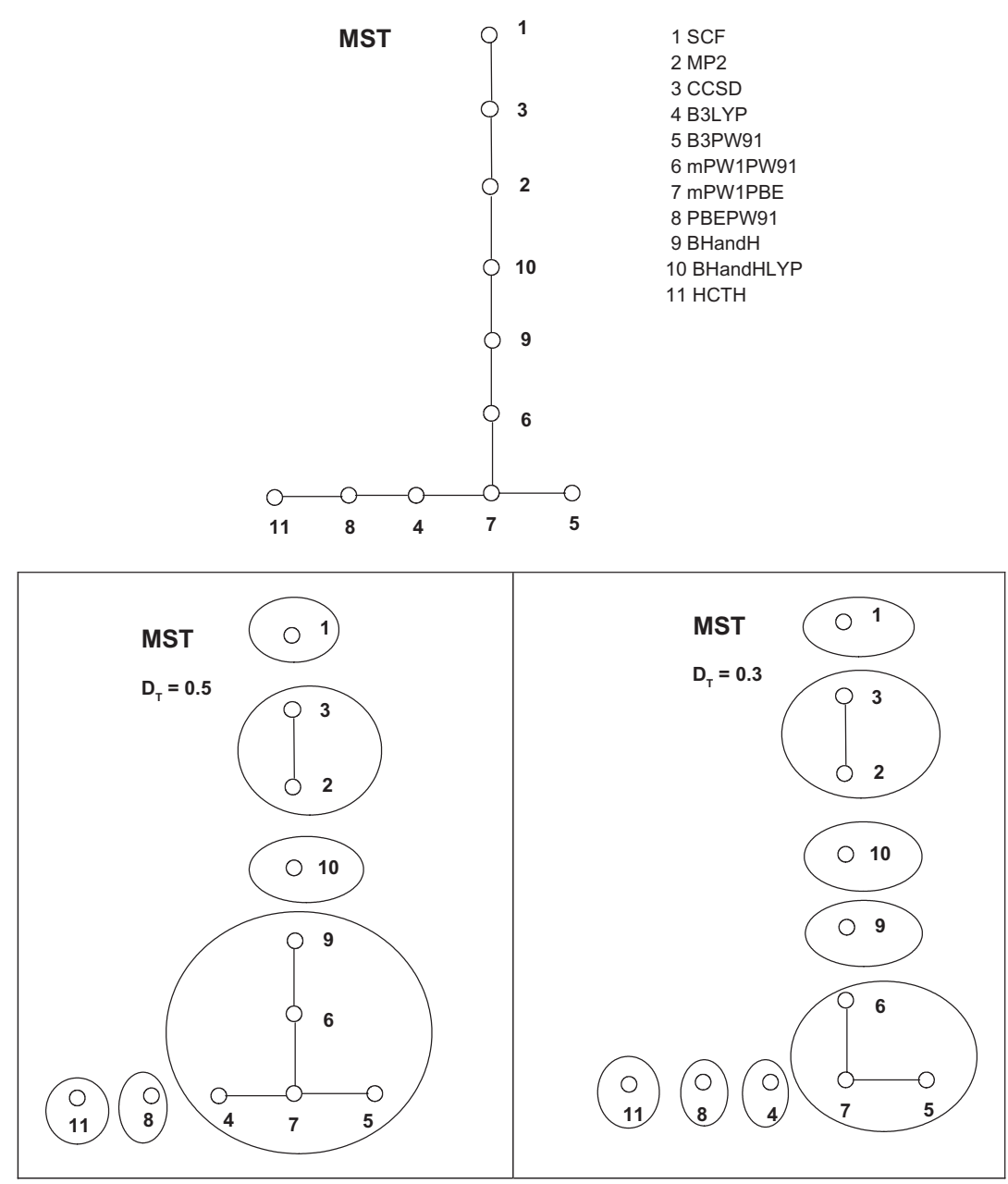

Figure 4 Minimum spanning tree (MST) and subsequent clustering of the space of theoretical descriptions.

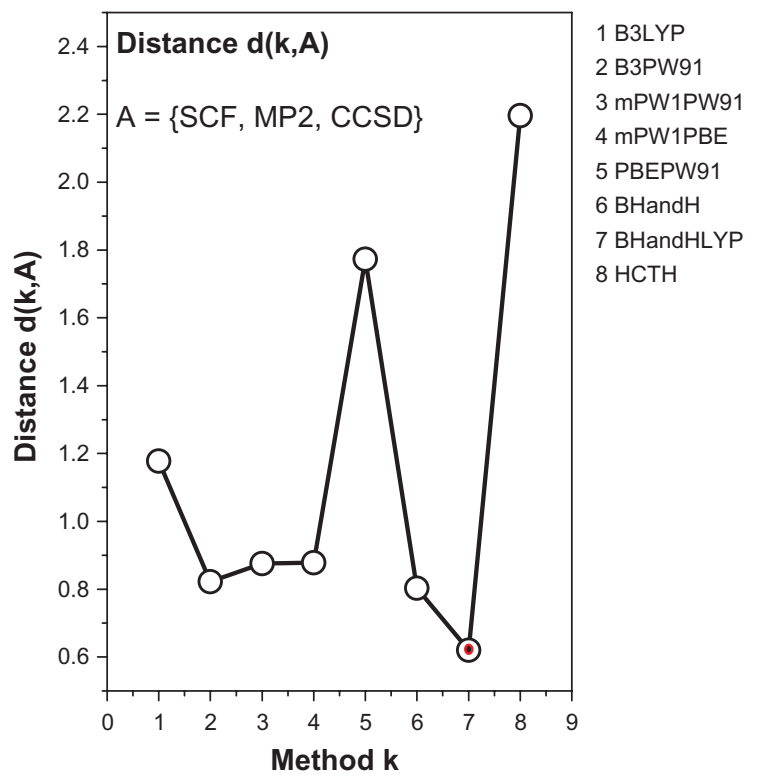

Figure 5 DFT method $(k)$ dependence of the distance $d(k, A)$ where $A=(S C F, M P 2, C C S D)$. The minimum occurs for the BHandLYP method, $\min _{k} d(k, A)=d(B H a n d L Y P, A)$. where:

DFT $=($ B3LYP, B3PW91, mPW1PW91, mPW1PBE, PBEPW91, BHandH, BHandHLYP, HCTH).

\section{Conclusion}

We have presented a quantitative analysis of the performance of ab initio and DFT methods over the electric properties of the important $\mathrm{NaCl}$ diatomic molecule. Our approach brings forth fundamental characteristics of the DFT methods, not easily obtainable in a conventional presentation. The DFT method most similar to presumably the most accurate CCSD one is BHandHLYP. A quantification of the similarity of the individual DFT methods to the subset (SCF, MP2, CCSD) shows that most similar is BHandHLYP followed by $\mathrm{BHandH}$. The most distant or least similar DFT method is $\mathrm{HCTH}$ :

$$
\mathrm{D}(\mathrm{HCTH}, \mathrm{A})>\underset{\mathrm{k} \in \mathrm{DFT}}{\mathrm{D}(\mathrm{k}, \mathrm{A})}>\mathrm{D}(\mathrm{BHandHLYP}, \mathrm{A})
$$


where:

$\mathrm{A}=(\mathrm{SCF}, \mathrm{MP} 2, \mathrm{CCSD})$

DFT $=$ (B3LYP, B3PW91, mPW1PW91, mPW1PBE, PBEPW91, BHandH, BHandHLYP, HCTH).

\section{Disclosure}

The author reports no conflicts of interest in this work.

\section{References}

1. Jones RO, Gunnarson O. The density functional formalism, its applications and prospects. Rev Mod Phys. 1989;61:689-746.

2. Civalleri B, Presti D, Dovesi R, Savin A. On choosing the best density functional approximation. Chem Modell. 2012;9:168-185.

3. Scheiner AC, Baker J, Andzelm JW. Molecular Energies and Properties from Density Functional Theory: Exploring Basis Set Dependence of Kohn-Sham Equation Using Several Density. Functionals. Int J Quant Chem. 1997;18:775-795.

4. Cai ZL, Sendt K, Reimers JR. Failure of density-functional theory and time-dependent density-functional theory for large extended $\mathrm{p}$ systems. J Chem Phys. 2002;117:5543-5549.

5. del Campo JM, Gázquez JL, Trickey SB, Vela A. Non-empirical improvement of PBE and its hybrid PBE0 for general description of molecular properties. J Chem Phys. 2012;136(10):104108.

6. Maroulis G. Evaluating the Performance of Correlated Methods in Molecular Property Calculations: Pattern Recognition and Clustering in Spaces of Theoretical Descriptions. Int J Quant Chem. 1995;55: $173-180$.

7. Maroulis G. On the accurate theoretical determination of the static hyperpolarizability of trans-butadiene. J Chem Phys. 1999;111:583-591.

8. Maroulis M. J Chem Phys. 2000;113:1813-1820.

9. Christodouleas C, Xenides D, Simos TE. J Comput Chem. 2010;31: $412-420$.

10. Xenides D, Karamanis P, Pouchan C. A critical analysis of the performance of new generation functionals on the calculation of the (hyper) polarizabilities of clusters of varying stoichiometry: Test case the $\mathrm{Si}_{\mathrm{m}} \mathrm{Ge}_{\mathrm{n}}$ $(\mathrm{m}+\mathrm{n}=7, \mathrm{n}=0-7)$ clusters. Chem Phys Lett. 2010;498: 134-139.

11. Johnson MD, Subbaswamy KR, Senatore G . Hyperpolarizabilities of alkali halide crystals using the local-density approximation. Phys Rev $B$. 1987;36:9202-9211.

12. Ching WY, Gan F and Huang MZ. Band theory of linear and nonlinear susceptibilities of some binary ionic insulators. Phys Rev B 1995;52: 1596-1611.

13. Guella T, Miller TM, Stockdale JAD, Bederson B, Vušković L. J Chem Phys. 1991;94:6857-6861.

14. Chauhan RS, Sharma SC, Sharma SB, Sharma BS. Analysis of polarizabilities, potentials, and geometries of alkali-halide dimers. $J$ Chem Phys. 1991;95:4397-4406.

15. Weis P, Ochsenfeld C, Ahlrichs R, Kappes MM. Ab initio studies of small sodium-sodium halide clusters, $\mathrm{Na}_{\mathrm{n}} \mathrm{CI}_{\mathrm{n}}$ and $\mathrm{Na}_{\mathrm{n}} \mathrm{CI}_{\mathrm{n}-1},(\mathrm{n} \leq 4) . J$ Chem Phys. 1992;97:2553-2560.

16. Maroulis G, Thakkar AJ. Multipole moments, polarizabilities, and hyperpolarizabilities for $\mathrm{N}_{2}$ from fourth-order, many-body perturbation theory calculations. J Chem Phys. 1988;88:7623-7632.

Reports in Theoretical Chemistry

\section{Publish your work in this journal}

Reports in Theoretical Chemistry is an international, peer-reviewed, open access journal publishing original research, reports, reviews and commentaries on all areas of theoretical chemistry. The manuscript management system is completely online and includes a very quick and fair
17. Maroulis G. A systematic study of basis set, electron correlation, and geometry effects on the electric multipole moments, polarizability, and hyperpolarizability of HCl. J Chem Phys. 1998;108:5432-5448.

18. Maroulis G. Computational Aspects of Interaction Hyperpolarizability Calculations. A Study on $\mathrm{H}_{2} \ldots \mathrm{H}_{2}, \mathrm{Ne} \ldots \mathrm{HF}, \mathrm{Ne} \ldots \mathrm{FH}, \mathrm{He} \ldots \mathrm{He}, \mathrm{Ne} \ldots$ $\mathrm{Ne}$, Ar...Ar, and Kr...Kr. J Phys Chem A. 2000;104:4772-4779.

19. Maroulis G. Accurate electric multipole moment, static polarizability and hyperpolarizability derivatives for $\mathrm{N}_{2}$. J Chem Phys. 2003;118: 2673-2687.

20. Haskopoulos A, Maroulis G. Interaction induced electric dipole moment and (hyper)polarizability in the dihydrogen-helium pair. Chem Phys. 2010;367:127-135.

21. Buckingham AD. Permanent and induced molecular moments and long-range intermolecular forces. Adv Chem Phys. 1967;12:107-142.

22. McLean AD, Yoshimine M. Theory of molecular polarizabilities. J Chem Phys. 1967;47:1927-1935.

23. Pullman B. Intermolecular interactions : From diatomics to biopolymers. New York: Wiley. 1978: p.1

24. Szabo A, Ostlund NS. Modern Quantum Chemistry. New York: McMillan; 1982.

25. Wilson S. Electron correlation in molecules. Oxford: Clarendon; 1984.

26. Helgaker T, Jørgensen P, J.Olsen J. Molecular Electronic-Structure Theory. Chichester: Wiley; 2000.

27. Frisch MJ, Trucks GW, Schlegel HB, et al. GAUSSIAN 03, Revision D.01. Wallingford (CT): Gaussian; 2004.

28. Maroulis G. Applying Conventional Ab Initio and Density Functional Theory Approaches to Electric Property Calculations. Quantitative Aspects and Perspectives. Struct Bond. 2012;149:95-130

29. Chartrand G, Lesniak L. Graphs and digraphs. Belmont (CA): Wadsworth; 1986.

30. Spath H. Cluster analysis algorithms. Chichester, UK: Ellis Horwood; 1980.

31. Woon DE, Dunning Jr TH. Gaussian basis sets for use in correlated molecular calculations. III. The atoms aluminum through argon. J Chem Phys. 1993;98:1358-1371.

32. Huber KP, G.Herzberg G. Molecular spectra and molecular structure: IV. Constants of diatomic Molecules. New York: Van Nostrand; 1979.

33. Matcha, RL. Theoretical Analysis of the Electronic Structure and Molecular Properties of the Alkali Halides. III. Sodium Chloride. J Chem Phys. 1968;48:335-343.

34. Backskay GB, Buckingham AD. Theoretical Analysis of the Electronic Structure and Molecular Properties of the Alkali Halides. III. Sodium Chloride. Mol Phys.

35. Pluta T. Nonlinear electric properties of alkali metal halides. Mol Phys. 2001;99:1535-1547.

36. Hebert AJ, Lovas FJ, Melendres CA, Hollowell CD, Story TL Jr, Street Jr K. Dipole Moments of Some Alkali Halide Molecules by the Molecular Beam Electric Resonance Method. J Chem Phys. 1968;48: 2824-2825.

37. Maroulis G, Makris C, Xenides D, Karamanis P. Electric dipole and quadrupole moment and dipole polarizability of CS, SiO and SiS. Mol Phys. 2000;98:481-491.

peer-review system. Visit http://www.dovepress.com/testimonials.php to read real quotes from published authors. 